\title{
Electron Density Measurement by Raman Calibration of the HuanLiuqi-2A Thomson Scattering System
}

\author{
Chunhua LIU, Yuan HUANG, Zhen FENG, Ke YAO, Lin NIE，Yuqin WANG，Jie SHANG, \\ Yi LIU, Zhongbing SHI, Qingwei YANG and Xuru DUAN \\ Southwestern Institute of Physics, P.O. Box 432, Chengdu, Sichuan 610041, China
}

(Received 3 December 2013 / Accepted 3 March 2014)

\begin{abstract}
Thomson scattering system is absolutely calibrated by anti-Stokes rotational Raman scattering from nitrogen gas filled in HuanLiuqi-2A (commonly referred to as HL-2A) chamber. Since the intensity of strayed laser light from the lower and upper divertor throats is very strong, additional transimission-type short pass filters are directly piled up on each interference filter to block the laser wavelength. The obtained data of electron density show good reliability, and they are in time trace coincident with the measured results of HCN laser interferometer. The uncertainties of measured results and the improving methods of the system are discussed.
\end{abstract}

(C) 2014 The Japan Society of Plasma Science and Nuclear Fusion Research

Keywords: Raman scattering, Thomson scattering, electron density calibration, HL-2A tokamak

DOI: $10.1585 /$ pfr.9.1402042

\section{Introduction}

The Thomson scattering (TS) is one of the most important diagnostic tools to measure electron temperature and density profiles in high temperature plasmas. The electron density results could be measured by rotational Raman scattering and Rayleigh scattering. Rayleigh scattering from neutral gas, such as a pure gas of Ar [1] or a mixture of air [2] has been reported to calibrate density measurements of Thomson scattering systems. Because there is no wavelength shift from Rayleigh scattering, a special wavelength channel should be equipped to detect the unshifted Rayleigh scattering lights. More importantly, the strayed laser light of the TS system must be suppressed at a lower level to increase the signal-to-noise ratio. These requirements imply that Rayleigh calibration is not available for the present core-HL-2A TS system, because the HL-2A tokamak has unique lower and upper closed divertors [3], in which the multi-pole coils are located close to each other and covered with water-flowing pipers embedded on baffles, as a result the throat passage is curved and very narrow. So the strayed laser lights of TS system on HL-2A are difficult to be actively suppressed.

The anti-Stokes rotational Raman scattering from nitrogen [4-9], hydrogen [10-13], deuterium [8, 9] and air [14] has been applied to obtain electron density data of Thomson scattering systems on many tokomak devices. With a wavelength shift caused by the rotational Raman scattering, it is easier to undertake density calibrations, even if the strayed laser light is very strong.

In this article, the present core-Thomson scattering system is briefly described in Sec. 2, the procedure of antiStokes rotational Raman scattering from nitrogen gas is

author'se-mail: liuchunhua@swip.ac.cn then introduced in Sec. 3, some examples of experiment results are presented in Sec. 4 , and the last is the summary and discussion.

\section{HL-2A Thomson Scattering System}

A single point TS measuring system [15], as shown in Fig. 1, is developed for HL-2A tokamak, in which a high energy Nd-doped yttrium aluminum garnet (Nd:YAG) laser is used. The Nd:YAG laser is composed of one oscillator and three stage amplifiers. Its main parameters are wavelength of $1064 \mathrm{~nm}$, pulse width of about $10 \mathrm{~ns}$, beam diameter of $30 \mathrm{~mm}$, pulse energy of $6 \mathrm{~J}$ at $1 \mathrm{~Hz}$ or $5 \mathrm{~J}$ at $5 \mathrm{~Hz}$ or $4 \mathrm{~J}$ at $10 \mathrm{~Hz}$ depending on different repetition rate, and $4 \mathrm{~J}$ at $10 \mathrm{~Hz}$ is the normal working conditions. The $\mathrm{Nd}$ :YAG laser beam is infrared and short pulsed, for its alignment, we use a visible green laser beam of $30 \mathrm{~mm}$ also in diameter and a 2-color filter, which highly reflects the green beam and transmits the infrared beam with $45^{\circ}$ incidence angle. The both two beams are adjusted to travel horizontally along the same axis and then delivered into input optics. At there, a mirror reflects them upwards and an $f=5 \mathrm{~m}$ lens focuses them into the vacuum chamber from the bottom to the top. Because the throat passage of HL-2A lower divertor is curved and very narrow, it is important to allow the YAG laser beam to traverse the machine without any risk to damage it. So behind the output window, a TV camera is used to monitor the position of the green beam, which is checked to be co-axis with the infrared laser beam. Though careful alignments are made for the laser beams, the strayed laser light is very strong and it is confirmed to be produced mainly from the lower and upper divertor throats when the YAG laser passing through. While in other TS systems [1,2], the strayed laser light is 


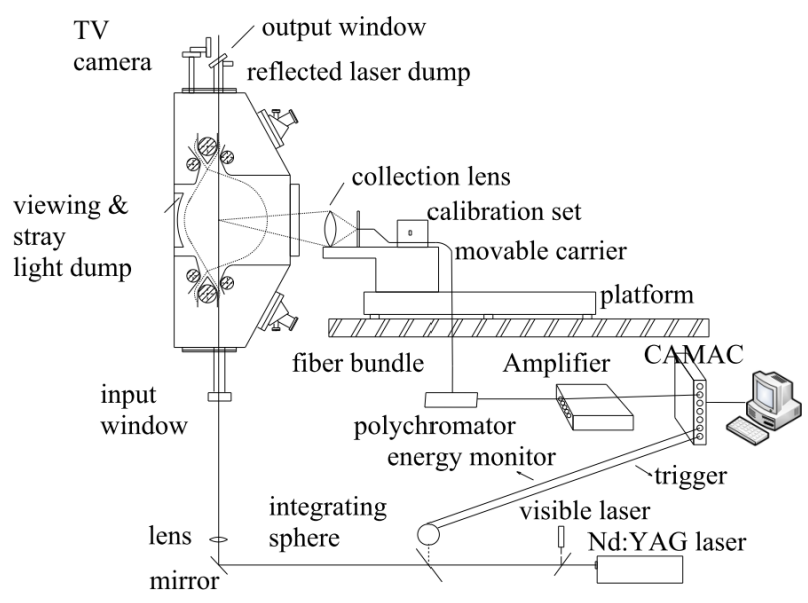

Fig. 1 Schematic layout of the Thomson scattering system on the HL-2A tokamak.

mainly produced from exit window and laser dump.

A 5-channel polychromator is used to separate scattered lights. The measured spectral responsibility includes the integrating effects of collection lens' transmission, fiber bundle's transmission, lens and filter's transmission of a polychromator, quantum efficiency and internal gain of a Si-APD detector, amplifier's gain, and digitizer's resolution, etc. Because the intensity of strayed laser light from the lower and upper divertor throats is very strong, additional transmission-type short pass filter has been directly piled up after each interference filter. The blocking ratios at $1064 \mathrm{~nm}$ of the short pass filter and interference filter are high up to $10^{-4}$ and $10^{-5}$, respectively. Thus the intensity of strayed laser light is passively suppressed and the Raman scattering signals from nitrogen gas are successfully obtained. The $\mathrm{CH} 1$ is used to detect Raman scattering lights in the calibration experiments.

\section{Raman Calibration}

The intensity of Thomson scattering signal in channel 1 is written as:

$$
\begin{aligned}
S^{T}= & E_{1} L \Delta \Omega n_{\mathrm{e}}\left(\mathrm{d} \sigma^{T} / \mathrm{d} \Omega\right) \\
& \times \int T_{\mathrm{w}}(\lambda) f_{\mathrm{CH} 1}(\lambda) S\left(\lambda, T_{\mathrm{e}}\right) \mathrm{d} \lambda,
\end{aligned}
$$

where, $n_{\mathrm{e}}$ is electron density, $T_{\mathrm{e}}$ is electron temperature in $\mathrm{eV}, E_{1}$ is the incident laser energy, $\Delta \Omega$ is the collecting solid angle, $L$ is the length of the scattering volume imaged into the polychromator entrance slit, $\mathrm{d} \sigma^{T} / \mathrm{d} \Omega$ is the differential cross section of Thomson scattering, $T_{\mathrm{w}}$ is the transmittance of the viewing window, $f_{\mathrm{CH} 1}$ is the responsibility of the first spectral channel as shown in Fig. $2, S\left(\lambda, T_{\mathrm{e}}\right)$ is the power function of Thomson scattering in the nun-collective regime, $\lambda$ is the scattered light wavelength in $\mathrm{nm}$. In the traditional $90^{\circ}$ scattering configuration and electron temperature in the range from $100 \mathrm{eV}$ to $100 \mathrm{keV}$, A.C. Selden gives the accurate expression of scattering spectrum [16]

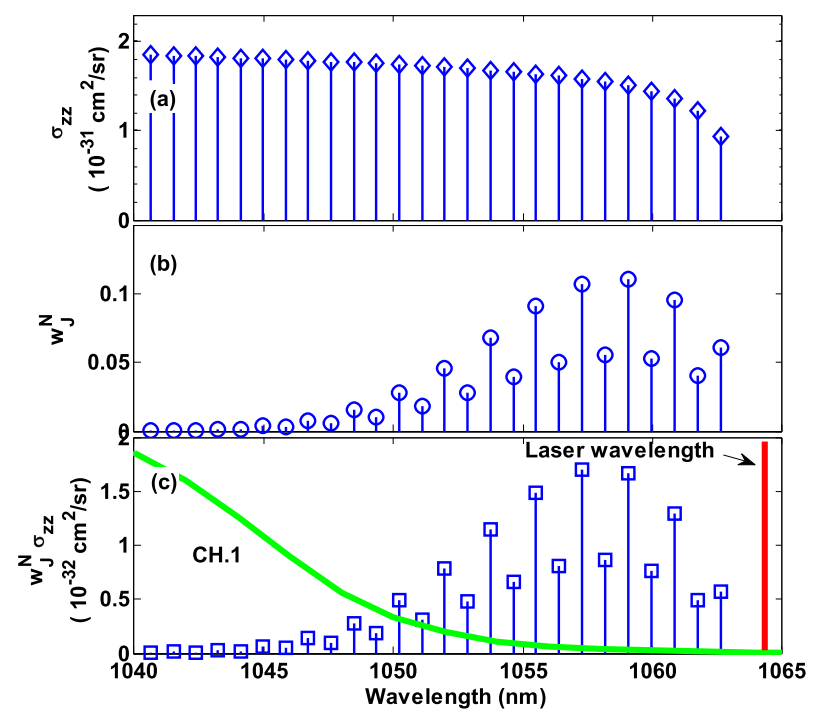

Fig. 2 The calculation results of (a) Raman cross sections for molecular nitrogen $\sigma_{z z} / s r$, (b) $w_{J}^{N}$, and (c) effective Raman scattering cross sections for molecular nitrogen $w_{J}^{N} \sigma_{z z} / s r$ and spectral responsibility of $\mathrm{CH} 1$, and laser wavelength $1064 \mathrm{~nm}$ is also marked.

with the relativistic effects:

$$
\begin{aligned}
S\left(\lambda, T_{\mathrm{e}}\right)= & {\left[1+1.875 b^{-1}+2.695 b^{-2}\right] } \\
& \times \frac{C\left(T_{\mathrm{e}}\right) \exp \left[b\left(T_{\mathrm{e}}\right) X(\theta, \eta)\right]}{A(\theta, \eta)},
\end{aligned}
$$

in which,

$$
\begin{aligned}
& b\left(T_{\mathrm{e}}\right)=-5.11 \times 10^{5} / T_{\mathrm{e}}, \\
& \mathrm{C}\left(T_{\mathrm{e}}\right)=2.86 \times 10^{2} /\left(\lambda_{\mathrm{l}} \sqrt{T_{\mathrm{e}}}\right), \\
& A(\theta, \eta)=(1+\eta)^{3} \sqrt{2(1-\cos \theta)(1+\eta)+\eta^{2}}, \\
& X(\theta, \eta)=\sqrt{1+\eta^{2} /[2(1-\cos \theta)(1+\eta)]}-1,
\end{aligned}
$$

$\theta$ is the scattering angle, $\lambda_{1}$ is the incident laser wavelength, $\eta=\left(\lambda-\lambda_{1}\right) / \lambda_{1}$.

As to anti-Stokes rotational Raman scattering, the rotational transition rule is $J \rightarrow J-2$ and the scattered light is corresponding to a line spectrum, a similar function could be given for the intensity of Raman scattering signal:

$$
S^{R}=E_{1} L \Delta \Omega n^{N} \sum_{J=2}^{\infty} w_{J}^{N} \frac{\mathrm{d} \sigma_{J}^{N}}{\mathrm{~d} \Omega} f_{\mathrm{CH} 1}\left(\lambda_{J}^{N}\right) .
$$

Where, $N$ and $J$ mean nitrogen gas and the initial rotational-angular-momentum number, respectively. $n^{N}$ stands for the number density of nitrogen molecules, $n_{J}^{N}$ is the molecule density in the initial upper state $J$ and $w_{J}^{N}=n_{J}^{N} / n^{N}$ is the corresponding fraction. $\lambda_{J}$ is the wavelength of the Raman scattering light, it can be calculated from the wavenumber shift, $\delta v=\left(4 B_{0}-14 D_{0}\right)(J-0.5)$, with known rotational constants $B_{0}$ and $D_{0}$ for the lowest vibrational level. For a gas in thermal equilibrium at gas temperature $T$,

$$
w_{J}^{N}=W^{-1} g_{J}(2 J+1) \exp \left[-E_{J} / k T\right],
$$


$g_{J}$ is the statistical weight factor depending on the nuclear spin quantum number, $I . E_{J}=J(J+1) h c B_{0}$ is the rotational energy, the gas temperature is taken as room temperature in the experiments, $T=295 \mathrm{~K}$. For nitrogen gas, $B_{0}=1.99 \mathrm{~cm}^{-1}, D_{0}=5.74 \times 10^{-6} \mathrm{~cm}^{-1}, g_{J}$ is 6 when $J$ even and 3 when $J$ odd, $I=1$. $W$ is a factor determined by the normalization condition, $\sum_{J=0}^{\infty} w_{J}^{N}=1$, and it could be calculated by $[17,18]$,

$$
W \approx \frac{(2 I+1)^{2} k T}{2 h c B_{0}}
$$

$h$ is Planck's constant, and $c$ is the speed of light in vacuum, $k$ is Boltzmann constant. For nitrogen gas at $T=$ $295 \mathrm{~K}, W=463.11$. The Raman wavelength of scattered line spectrum is given by [19],

$$
\lambda_{J} \approx \lambda_{1}-4 \lambda_{1}^{2} B_{0}(J-0.5),
$$

$\mathrm{d} \sigma_{J}^{N} / \mathrm{d} \Omega$ is the differential cross section of the antiStokes rotational Raman transition and it could be expressed as,

$$
\begin{aligned}
& \frac{\mathrm{d} \sigma_{J}^{N}}{\mathrm{~d} \Omega}=\sigma_{z z}\left[(1-\rho) \cos ^{2} \psi+\rho\right], \\
& \sigma_{z z}=\frac{64 \pi^{4}}{45} \frac{3 J(J-1)}{2(2 J+1)(2 J-1)} \frac{\gamma^{2}}{\lambda_{J}^{4}} .
\end{aligned}
$$

$\psi$ is the angle between the electric-field polarization directions of the incident and observed scattered lights, $\psi=90^{\circ}$ at our case. In conveniently defined right-angle scattering configuration, the directions of polarization and propagation of the linearly polarized incident beam are in the $y$ direction and $z$ direction, the scattered light is observed in the $x$ direction. The cross section $\sigma_{z z}$ is proportional to the content of scattered light polarized in the $z$ direction, which is parallel to the incident-beam polarization. The depolarization factor $\rho$ is the ratio of the perpendicular component to the parallel component. The Ramanscattered light is largely depolarized, and the theoretical value of $\rho$ is 3/4. $\gamma$ is the value of polarizability anisotropy, it is estimated to be $0.66 \times 10^{-24} \mathrm{~cm}^{3}$ at the incident wavelength of $1064 \mathrm{~nm}$ by linear extrapolation $[20,21]$ from published data, while $\gamma$ is experimentally found [4] to be about $0.71 \times 10^{-24} \mathrm{~cm}^{3}$ at $1064 \mathrm{~nm}$.

Figure 2 gives the spectral responsibility of channel 1 and effective cross section distribution of rotational Raman lines for nitrogen molecules. It is clear that the Raman scattering signals could be partially detected by channel 1 in the polychromator. If the nitrogen gas density is monitored during Raman scattering experiments, the electron density could be calculated according to the Eq. (1) and Eq. (3).

In the procedure of Raman calibration experiments, nitrogen gas is filled into the vacuum vessel of HL-2A tokomak, and Raman signal intensities are measured at six pressure values from $5 \mathrm{kPa}$ to $45 \mathrm{kPa}$ to determine the values of $n^{N} / S^{R}$. The pressure values of nitrogen gas

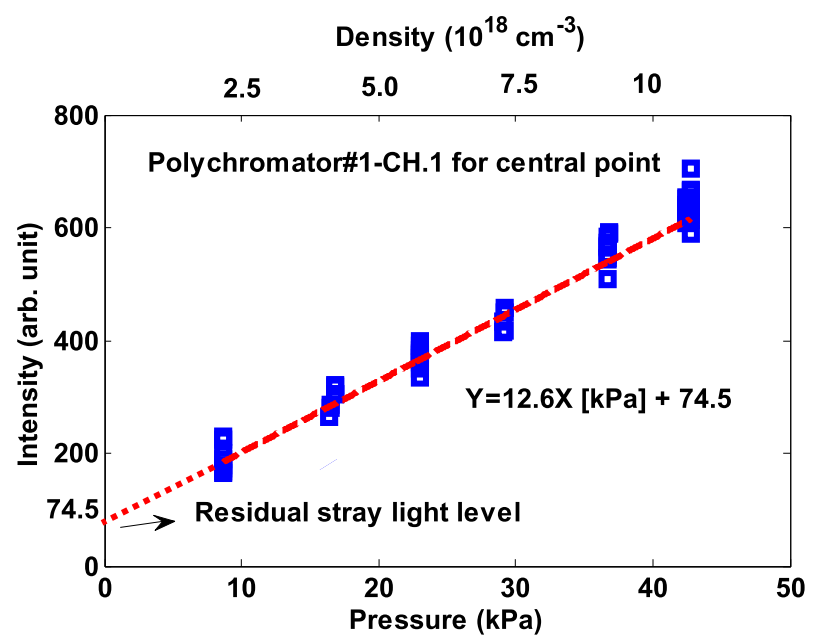

Fig. 3 Total intensity measured in polychromator \#1-CH.1 with different pressure values of nitrogen gas. The calibration factor is defined by the fitting line.

are monitored with a capacitance manometer, which is installed on the HL-2A tokamak. The density dependence of Raman scattering signals detected by channel 1 is shown in Fig. 3. In order to decrease the effect of the dust in the vacuum vessel on the Raman scattering signals, about two hours later after filling nitrogen gas the TS system begin to collect signals. The data from about 10 shots (100 laser pulses) were accumulated to decrease the statistical uncertainty. Another worth-noting point is the phenomena of spark discharge excited by high energy laser. The threshold value of electric breakdown of nitrogen gas is inversely proportional to the quadratic pressure [22] or density. If the pressure is too high and the laser power is intense enough, the phenomena of spark discharge are easy to occur and to disturb the Raman scattering signals. With consideration of the two points above, the experimental results obtained are that the signal density is proportional to the nitrogen pressure. The calibration coefficient of the TS system is obtained from the gradient by fitting the measured data of total intensity $v s$ pressure into a line with least square method, as shown in Fig. 3, the fitted line is expressed as $Y=12.6 X[\mathrm{kPa}]+74.5$, where $X$ is the gas pressure in $\mathrm{kPa}$ and $Y$ is the signal intensity. The residual stray light level can be estimated as $Y=74.5$ when $X=0$, which would be the total intensity when the gas pressure value is close to zero. As we can see, the residual stray light level is still high. The cause is that the throat passage of the HL-2A closed divertor is quite narrow and the strayed laser is so strong, the rejection ratio of filters in a polychromator is not high enough to suppress all the stray laser lights.

\section{Experimental Results of Electron Density}

The electron density of HL-2A plasma has been successfully measured with calibrated high energy YAG laser 


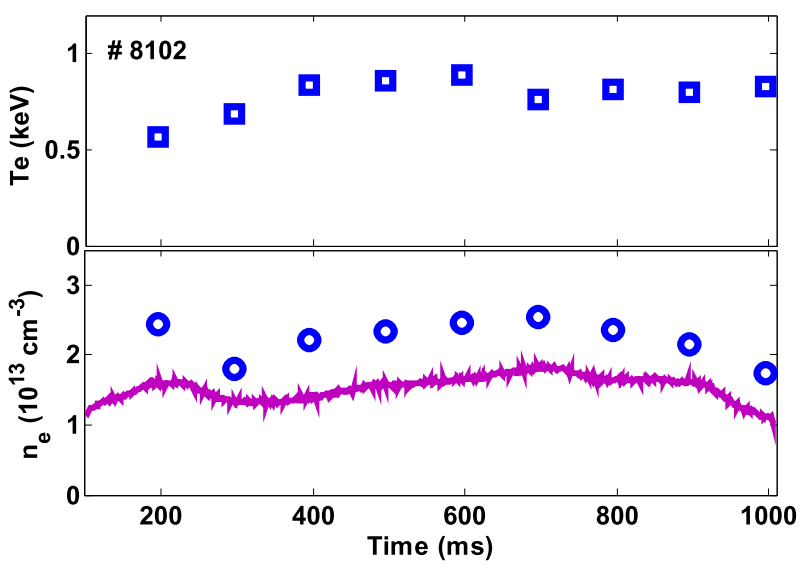

Fig. 4 Electron temperature and electron density measured by Thomson scattering system, line-averaged electron density measured by $\mathrm{HCN}$ laser interferometer. With the peaking factor of 1.3 1.5, the time trace of electron density by TS is consistent with that of line-averaged electron density by HCN.

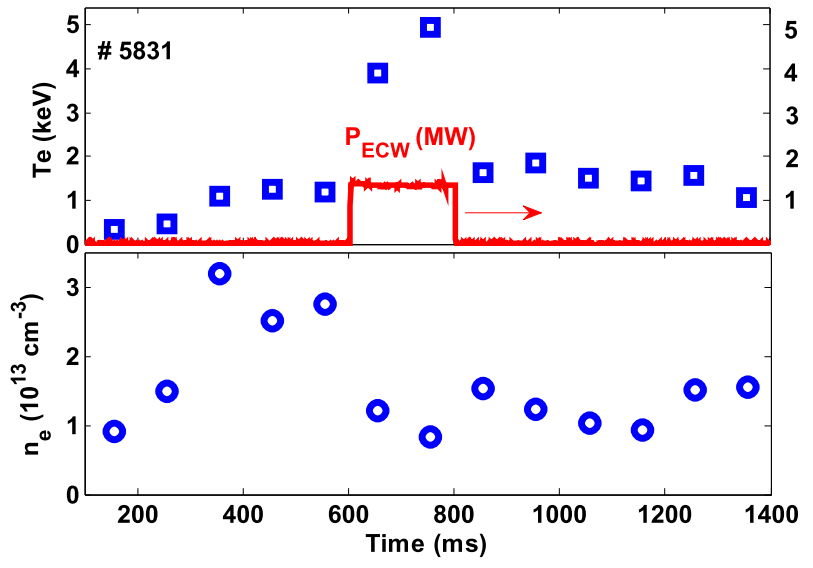

Fig. 5 Electron temperature and density measured by Thomson scattering system during powerful ECW heating.

Thomson scattering system. Figure 4 shows the measurement results of electron temperature and density, and chord-averaged density measured by hydrogen cyanide (HCN) laser interferometer [23]. In the shot \#8102, the plasma parameters are plasma current $I_{\mathrm{p}}=150 \mathrm{kA}$, magnetic field $B_{\mathrm{T}}=1.32 \mathrm{~T}$, and the plasma is heated by $68 \mathrm{GHz}$ electron cyclotron wave $(\mathrm{ECW})$ with power of $30 \mathrm{~kW}$ in the time range from $400 \mathrm{~ms}$ to $600 \mathrm{~ms}$. From the results we can see that the electron density measured by Thomson scattering has almost the same time tendency as that by $\mathrm{HCN}$ interferometer, and the former is about $1.3 \sim 1.5$ times higher than the latter, which is usually called as the density peaking factor. The results imply that the calibration coefficient with nitrogen gas is reasonable.

Figure 5 shows the electron temperature and density with $1.35 \mathrm{MW}$ ECW heating power during $600 \mathrm{~ms}$ and $800 \mathrm{~ms}$ in shot \#5831. The plasma parameters are

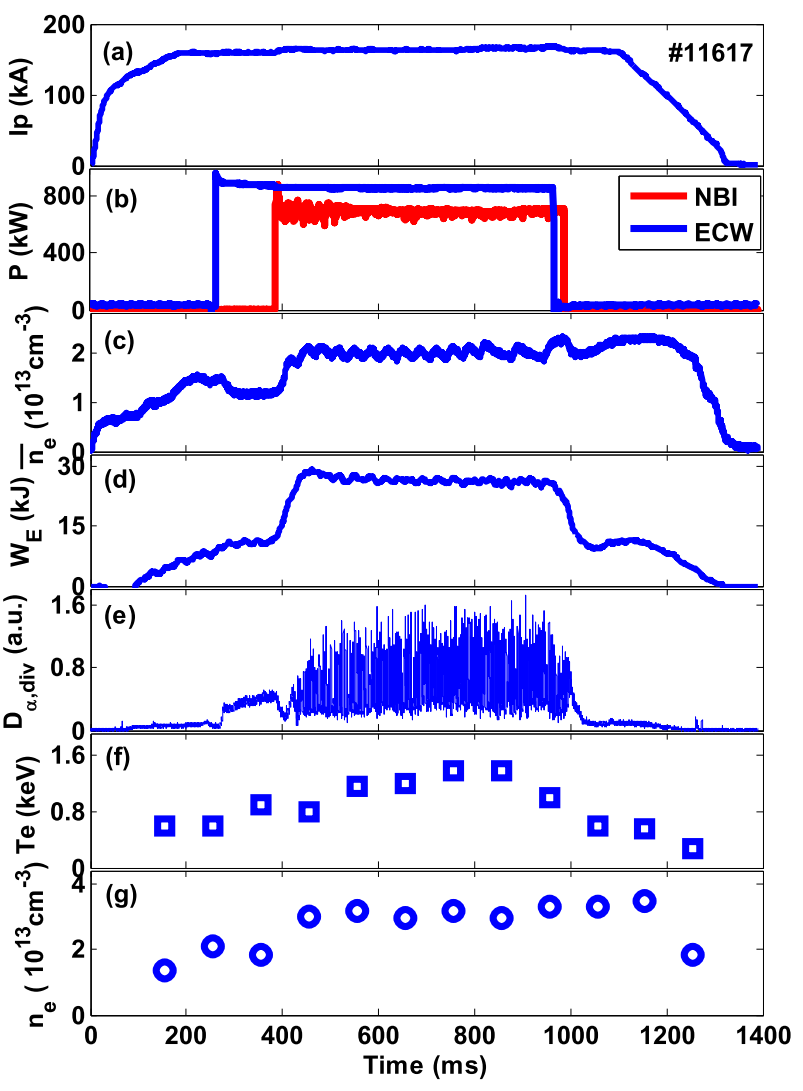

Fig. $6 \mathrm{H}$-mode discharge parameters in shot \#11617, and the results of electron temperature and density measured by Thomson scattering system. The time traces from top to down are the plasma current (a), the NBI and ECW heating power (b), the line-averaged density measured by $\mathrm{HCN}$ laser interferometer (c), the plasma stored energy (d), the $\mathrm{D} \alpha$ emissions in the divertor region (e), electron temperature (f) and electron density (g) measured by Thomson scattering system, respectively.

$I_{\mathrm{p}}=300 \mathrm{kA}, B_{\mathrm{T}}=2.4 \mathrm{~T}$. In the ECW heating range, electron temperature increases obviously, even up to $4.9 \mathrm{keV}$. On the contrary, electron density decreases seriously, even down to $1 \times 10^{13} \mathrm{~cm}^{-3}$, which is sometimes called as density pump-out phenomena caused by powerful ECW heating.

The calibration results of electron density are also analyzed under high confinement mode (H-mode) discharge conditions. An example of $\mathrm{H}$-mode discharge is shown in Fig. 6. Where, the time traces from top to down are the plasma current (a), the NBI and ECW heating power (b), the line-averaged density measured by $\mathrm{HCN}$ laser interferometer (c), the plasma stored energy (d), the D $\alpha$ emissions in the divertor region (e), electron temperature (f) and electron density (g) measured by Thomson scattering, respectively. The H-mode is terminated by the pulse duration of the NBI and ECW heating power. The ECW power of $850 \mathrm{~kW}$ is input at $t=260 \mathrm{~ms}$, the plasma density slightly drops and the "pump-out" phenomena happen. The NBI power is applied near $t=385 \mathrm{~ms}$, and the $\mathrm{L}-\mathrm{H}$ transition 
occurs after about $50 \mathrm{~ms}$. The H-mode discharge sustains for about $560 \mathrm{~ms}$ until the auxiliary heating power is turned off. From the results of electron temperature and density measured by Thomson scattering system, as shown in Figs. 6 (f) and (g), the electron temperature increases up to $1.5 \mathrm{keV}$ and the electron density keeps near $3 \times 10^{13} \mathrm{~cm}^{-3}$ during H-mode operation.

\section{Summary and Discussion}

The electron density of the HL-2A plasma in different conditions has been successfully measured with calibrated high energy Nd:YAG laser Thomson scattering system. The calibration results of electron density in L-mode and $\mathrm{H}$-mode discharges shows good repeatability, and the results measured by the system have given important messages on the physics properties of the high temperature plasma.

According to the Fig. 3, intensity of the residual stray light signal is 74.5. By subtracting the stray light signals, the ratios of the Raman scattering signals computed from the fitting line, at three different pressure values $P_{1}=8.7 \mathrm{kPa}, P_{3}=23 \mathrm{kPa}$ and $P_{4}=29.2 \mathrm{kPa}$ for $\mathrm{CH} 1$ are estimated as,

$$
\begin{aligned}
& \frac{P_{3}}{P_{1}}=2.64, \frac{S_{1}^{R}\left(P_{3}\right)}{S_{1}^{R}\left(P_{1}\right)}=2.64 \sim \frac{P_{3}}{P_{1}}, \\
& \frac{P_{4}}{P_{1}}=3.35, \frac{S_{1}^{R}\left(P_{4}\right)}{S_{1}^{R}\left(P_{1}\right)}=3.36 \sim \frac{P_{4}}{P_{1}} .
\end{aligned}
$$

It means the computed Raman intensity is proportional to the gas pressure, and the measured data of electron density are in good agreement with the expected values. Most of the experimental uncertainty was due to the variance of laser energy which would be monitored with more confidence in future and the shot noise of lights accepted by the Si-APD detector. The lab temperature also has much influence on properties of Si-APD noise and its gain, as well as the transmittance of a filer. Many antiStokes Raman lines of nitrogen gas located in the edge slope range of $\mathrm{CH} 1$ optical band-filter, varying temperature may seriously change the wavelength-transmittance in the slope of the band-filter, as a result the signal of Raman scattered lights was impacted. From this point of view, Raman calibration in $\mathrm{H}_{2}$ or $\mathrm{D}_{2}$ gas reduces the strong dependency of calibration accuracy on the filter transmission function, because there are only two strong anti-Stokes lines corresponding to the transitions $J=3 \rightarrow 1$ and $J=2 \rightarrow 0$. Considering $B_{0}\left(H_{2}\right)=59.34 \mathrm{~cm}^{-1}$ and $B_{0}\left(D_{2}\right)=29.91 \mathrm{~cm}^{-1}$, the corresponding Raman lines are $\lambda_{2-0}\left(H_{2}\right)=1023.69 \mathrm{~nm}$ and $\lambda_{3-1}\left(H_{2}\right)=996.82 \mathrm{~nm}$, $\lambda_{2-0}\left(D_{2}\right)=1043.68 \mathrm{~nm}$ and $\lambda_{3-1}\left(D_{2}\right)=1030.14 \mathrm{~nm}$, respectively. These lines have the advantage of far from the laser wavelength. But practically it is not recommended for the safety consideration that hydrogenic gases are explosive when they are pumped into air. Other uncertainties are the value of $\gamma$ used to calculate the Raman cross-sections, the transmittance of the viewing window and the pointing stability of the Nd:YAG laser beam. We just simply assume that the transmittance of the viewing window keeps unchanged during HL-2A experiments. Plasma discharge, glow-discharge cleaning and wall conditioning could put some kinds of coatings on the first surfaces of the optical windows. Those coatings can decrease the transmittance of the viewing window and make it vary with different wavelength. Fast Raman calibration may be needed for the TS system during HL-2A experiments, and not impacting the wall conditions and plasma discharges. The transit digitizers will also be used to differentiate the Raman or Thomson signals from stray laser light signals, and it will be helpful to decrease the data error. With the improvements on hardware for Raman scattering experiments, the edge-Thomson scattering system will also be calibrated to obtain the electron density profiles at HL-2A plasma edge during $\mathrm{H}$-mode discharges.

\section{Acknowledgments}

The authors acknowledge the contributions of P. $\mathrm{Lu}$ and B.Z. Fu. The authors also appreciate the useful discussions with I. Yamada, K. Gibson and C. Hsieh. This work is partially supported by the Chinese National Magnetic Confinement Fusion Science Program under Grants No. 2009GB107002, No. 2013 GB104002 and No. 2013 GB107002.

[1] D.G. Nilson et al., Rev. Sci. Instrum. 68, 704 (1997).

[2] J.H. Lee et al., Rev. Sci. Instrum. 81, 10D528 (2010).

[3] Y. Liu et al, Nucl. Fusion 45, S239 (2005).

[4] B.P. LeBlanc, Rev. Sci. Instrum. 79, 10E737 (2008).

[5] I. Yamada et al., Plasma Fusion Res. 2, S1106 (2007).

[6] J. Howard et al., J. Phys. D 12, 1435 (1979).

[7] J.M. de Regt et al., Rev. Sci. Instrum. 66, 3228 (1995).

[8] J. Howard et al., J. Phys. D: Appl. Phys. 12, 1435 (1979).

[9] L.A. Berni et al., Rev. Sci. Instrum. 74, 1200 (2003).

[10] T. Yamauchi and I. Yanagisawa, Appl. Opt. 24, 700 (1985).

[11] H. Röhr, Phys. Lett. A 81, 451 (1981).

[12] J.W. Hughes et al., Rev. Sci. Instrum. 74, 1667 (2003).

[13] F. Orsitto et al., Rev. Sci. Instrum. 66, 1167 (1995).

[14] I. Yamada et al., Rev. Sci. Instrum. 74, 1675 (2003).

[15] Y. Huang et al., Rev. Sci. Instrum. 78, 113501 (2007).

[16] A.C. Selden, Phys. Lett. A 79, 405 (1980).

[17] C.M. Penney et al., Opt. Soc. Am. 64, 712 (1974).

[18] G. Herzberg, Molecular spectra and molecular structure, volume I. Spectra of Diatomic Molecules (D. van Nostrand Company, Princeton, Second edition 1950).

[19] R. Scannell et al., Rev. Sci. Instrum. 81, 045107 (2010).

[20] M.J. van de Sande, "Laser scattering on low temperature plasmas-high resolution and stray light rejection," Ph.D. thesis, Technische Universiteit Eindhoven, 2002.

[21] K. Narihara, Fusion Eng. Des. 34, 67 (1997).

[22] Z.J. Jiang, "The study of ionized atmosphere with the intense laser," Master thesis, Changchun University of Science and Technology, 2008 [in Chinese].

[23] Y. Zhou et al., Rev. Sci. Instrum. 78, 113503 (2007). 